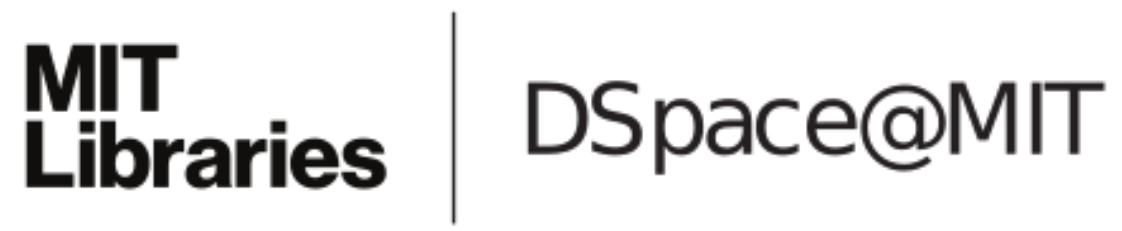

\author{
MIT Open Access Articles
}

Diquark correlations in a hadron from lattice $Q C D$

The MIT Faculty has made this article openly available. Please share how this access benefits you. Your story matters.

Citation: Green, Jeremy, Michael Engelhardt, John Negele, and Patrick Varilly. "Diquark correlations in a hadron from lattice QCD." In 19th Particles and Nuclei International Conference: PANIC 11, Cambridge, Massachusetts, USA, 24-29 July 2011, edited by Stephen G. Steadman, George S.F. Stephans, Frank E. Taylor. (AIP conference proceedings. High energy physics; 1441), AIP, 2012. (c) 2012 American Institute of Physics.

As Published: http://dx.doi.org/10.1063/1.3700503

Publisher: American Institute of Physics

Persistent URL: http://hdl.handle.net/1721.1/78661

Version: Final published version: final published article, as it appeared in a journal, conference proceedings, or other formally published context

Terms of Use: Article is made available in accordance with the publisher's policy and may be subject to US copyright law. Please refer to the publisher's site for terms of use. 


\section{AIP $\mid$ Conference Proceedings}

\section{Diquark correlations in a hadron from lattice QCD}

Jeremy Green, Michael Engelhardt, John Negele, and Patrick Varilly

Citation: AIP Conf. Proc. 1441, 172 (2012); doi: 10.1063/1.3700503

View online: http://dx.doi.org/10.1063/1.3700503

View Table of Contents: http://proceedings.aip.org/dbt/dbt.jsp?KEY=APCPCS\&Volume=1441\&lssue $=1$

Published by the American Institute of Physics.

\section{Additional information on AIP Conf. Proc.}

Journal Homepage: http://proceedings.aip.org/

Journal Information: http://proceedings.aip.org/about/about_the_proceedings

Top downloads: http://proceedings.aip.org/dbt/most_downloaded.jsp?KEY=APCPCS

Information for Authors: http://proceedings.aip.org/authors/information_for_authors

- Article-level metrics now available 


\title{
Diquark Correlations in a Hadron From Lattice QCD
}

\author{
Jeremy Green*, Michael Engelhardt ${ }^{\dagger}$, John Negele* and Patrick Varilly** \\ ${ }^{*}$ Center for Theoretical Physics, Massachusetts Institute of Technology, Cambridge, MA 02139 \\ ${ }^{\dagger}$ Department of Physics, New Mexico State University, Las Cruces, NM 88003-0001 \\ ${ }^{* *}$ Department of Physics, University of California, Berkeley, Berkeley, CA 94720
}

\begin{abstract}
Using lattice QCD, a diquark can be studied in a gauge-invariant manner by binding it to a static quark in a heavylight-light hadron. We compute the simultaneous two-quark density of a diquark, including corrections for periodic boundary conditions. We define a correlation function to isolate the intrinsic correlations of the diquark and reduce the effects caused by the presence of the static quark. Away from the immediate vicinity of the static quark, the diquark has a consistent shape, with much stronger correlations seen in the good (scalar) diquark than in the bad (axial-vector) diquark. We present results for $m_{\pi}=293 \mathrm{MeV}$ as well as $m_{\pi}=940 \mathrm{MeV}$, and discuss the behavior as the pion mass changes.
\end{abstract}

Keywords: diquark, lattice QCD, hadron structure

PACS: 12.38 .Gc, $14.20 .-\mathrm{c}$

\section{INTRODUCTION}

Diquarks are two-quark systems whose collective behavior may elucidate many phenomena of strong interactions [1]. Notably, the properties of baryons are often explained using a quark-diquark description [2,3]. Diquarks are also important ingredients in models of exotic hadrons [4].

The simplest diquark interpolating operators are quark bilinears with spinor part $q^{T} C \Gamma q$. The favored combinations are color antitriplet, even parity [4]. These are divided into "good" and "bad" diquarks. The good diquarks, $q^{T} C \gamma_{5} q$, have spin 0 and are flavor antisymmetric due to fermion statistics. The bad diquarks, $q^{T} C \gamma_{i} q$, have spin 1 and are flavor symmetric.

Both one-gluon exchange in a quark model $[5,6]$ and instanton [7] models give a spin coupling energy proportional to $\vec{S}_{i} \cdot \vec{S}_{j}$, which favors the good diquark over the bad diquark. The strength of this coupling falls off with increasing quark masses. For the instanton model, the effective interaction has a flavor dependence that also favors the good diquark.

\section{CORRELATION FUNCTION}

Since diquarks are not color singlets, studying them within the framework of lattice QCD has been done by combining one with a third quark to form a color singlet. Diquark attractions result in spatial correlations between the two quarks in the diquark, which have been probed by computing a two-quark density [8] or a gauge-fixed wavefunction [9].

In this work, we combined a diquark, formed from degenerate $u$ and $d$ quarks, with a static quark $s$. We used the baryon interpolating operator $B=\varepsilon^{a b c}\left(u_{a}^{T} C \Gamma d_{b}\right) s_{c}$, taking $\Gamma=\gamma_{5}$ for the good diquark and $\Gamma=\gamma_{1}$ for the bad diquark, and calculated the single quark density and the simultaneous two-quark density:

$$
\rho_{1}(\mathbf{r}) \propto \frac{\left\langle 0\left|B\left(\mathbf{0}, t_{f}\right) J_{0}^{u}(\mathbf{r}, t) \bar{B}\left(\mathbf{0}, t_{i}\right)\right| 0\right\rangle}{\left\langle 0\left|B\left(\mathbf{0}, t_{f}\right) \bar{B}\left(\mathbf{0}, t_{i}\right)\right| 0\right\rangle}, \quad \rho_{2}\left(\mathbf{r}_{1}, \mathbf{r}_{2}\right) \propto \frac{\left\langle 0\left|B\left(\mathbf{0}, t_{f}\right) J_{0}^{u}\left(\mathbf{r}_{1}, t\right) J_{0}^{d}\left(\mathbf{r}_{2}, t\right) \bar{B}\left(\mathbf{0}, t_{i}\right)\right| 0\right\rangle}{\left\langle 0\left|B\left(\mathbf{0}, t_{f}\right) \bar{B}\left(\mathbf{0}, t_{i}\right)\right| 0\right\rangle},
$$

where $J_{\mu}^{f}=\bar{f} \gamma_{\mu} f$ is the local vector current.

In order to isolate the correlations caused by the diquark interaction and remove the effect of the static quark, we define the normalized correlation function:

$$
C\left(\mathbf{r}_{1}, \mathbf{r}_{2}\right)=\frac{\rho_{2}\left(\mathbf{r}_{1}, \mathbf{r}_{2}\right)-\rho_{1}\left(\mathbf{r}_{1}\right) \rho_{1}\left(\mathbf{r}_{2}\right)}{\rho_{1}\left(\mathbf{r}_{1}\right) \rho_{1}\left(\mathbf{r}_{2}\right)}
$$

19th Particles and Nuclei International Conference (PANIC11)

AIP Conf. Proc. 1441, 172-174 (2012); doi: 10.1063/1.3700503

(C) 2012 American Institute of Physics 978-0-7354-1035-0/ $\$ 30.00$ 

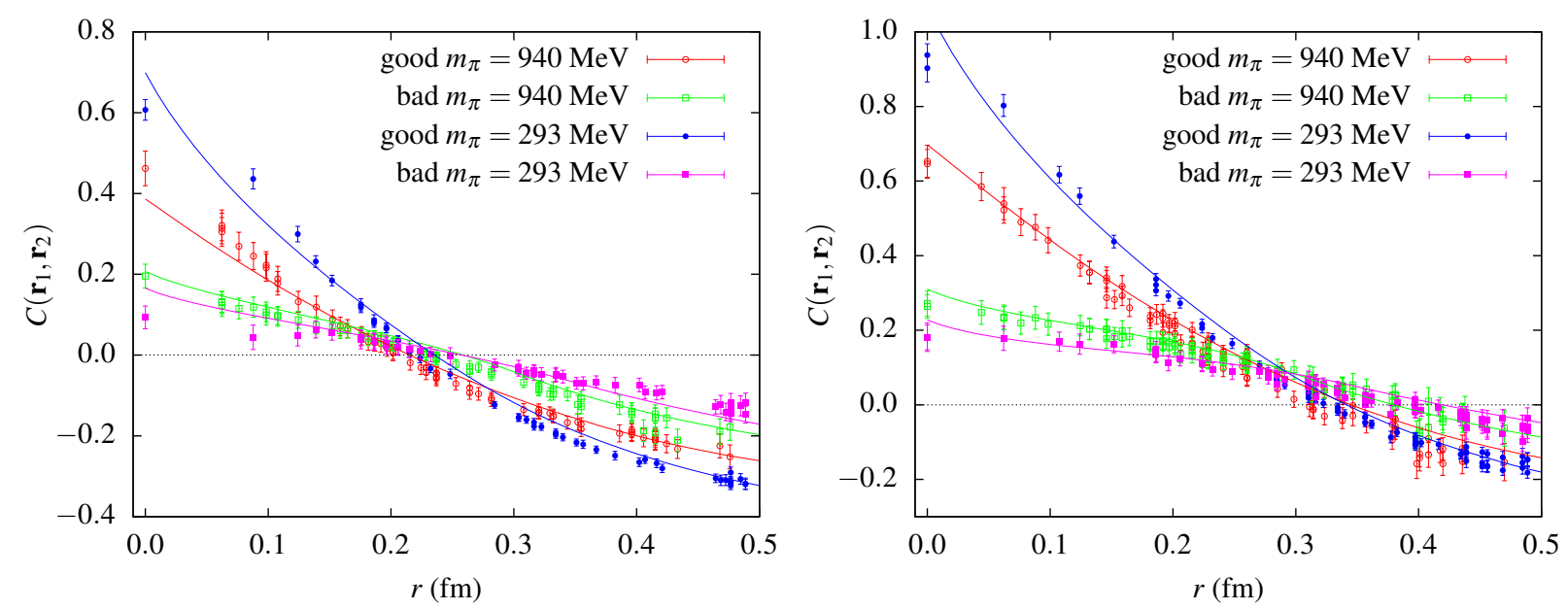

FIGURE 1. $C\left(\mathbf{r}_{1}, \mathbf{r}_{2}\right)$, as a function of $r$ (in fm) with $R=0.2 \mathrm{fm}$ (left) and $R=0.4 \mathrm{fm}$ (right), with $\mathbf{r} \perp \mathbf{R}$ for the good and bad diquarks and the two pion masses. The solid curves are derived from the fit to $\rho_{1,2}$.

This divides out the tendency to stay near the static quark and has the property of being zero if the two light quarks are uncorrelated (i.e. if $\rho_{2}\left(\mathbf{r}_{1}, \mathbf{r}_{2}\right)=\rho_{1}\left(\mathbf{r}_{1}\right) \rho_{1}\left(\mathbf{r}_{2}\right)$ ). The downsides are that $C$ does not integrate to zero (which would be the case without the denominator), and it is possible for $C(\mathbf{r}, \mathbf{r})$ to increase without bound as $|\mathbf{r}| \rightarrow \infty$.

\section{LATTICE CALCULATIONS}

We used a mixed action scheme [10] with domain wall valence quarks and staggered sea quarks, with $m_{\pi}=$ 293(1) MeV, lattice spacing $a=0.1241(25) \mathrm{fm}$, and volume $20^{3} \times 64$. For comparison, we also used a heavy quark mass, with $m_{\pi} \approx 940 \mathrm{MeV}$. Since the effects of dynamical sea quarks are negligible at that mass, we performed a calculation with quenched $\kappa=0.153$ Wilson fermions on the OSU_Q60a ensemble [11], which has volume $16^{3} \times 32$ with lattice spacing $a=0.088 \mathrm{fm}$.

In order to remove the contributions to $\rho_{1,2}$ caused by the periodic boundary conditions, we fitted an elevenparameter function (motivated by the so-called $\Delta$ ansatz for the static potential for interacting quarks at short distances $[12,13])$ to $\rho_{1,2}$ and used it to subtract off the nearest-neighbor image contributions. The image-corrected $\rho_{1,2}$ were then used to compute the correlation function $C$. A more detailed discussion is available in [14].

\section{RESULTS AND DISCUSSION}

We present results using the more convenient coordinates $\mathbf{R}=\left(\mathbf{r}_{1}+\mathbf{r}_{2}\right) / 2$ and $\mathbf{r}=\left(\mathbf{r}_{1}-\mathbf{r}_{2}\right) / 2$, labeling their magnitudes by $R=|\mathbf{R}|$ and $r=|\mathbf{r}|$, and using $\theta$ to denote the angle between $\mathbf{R}$ and $\mathbf{r}$.

As a check of how well the correlation function isolates the diquark from the effect of the static quark, we compared different directions of $\mathbf{r}$. Even at $R=0.2 \mathrm{fm}, C$ was independent of the direction of $\mathbf{r}$, indicating that this correlation function works quite well.

Finally, we can compare the systems. Figure 1 shows the profile of the correlation function $C$ in the direction $\mathbf{r} \perp \mathbf{R}$ at two fixed distances $R$ from the static quark to the center of the diquark, and Fig. 2 shows the full dependence of $C$ on $\mathbf{r}$, at fixed $R=0.4 \mathrm{fm}$. The good diquark has a large positive correlation at small $r$ that becomes negative at large $r$. The bad diquark has similar behavior with smaller magnitude. The difference between the good and bad diquarks is larger for the lighter pion mass, as expected from the quark mass dependence of the spin coupling that splits good and bad diquarks. As $R$ increases, both the correlation and the size of the positive region grow, although it is possible that some of this growth of $C$ as $R$ increases may arise from the normalization of the correlation function.

Our main conclusions are seen clearly in Fig. 2. The diquark correlations are highly independent of $\theta$, indicating negligible polarization by the heavy quark, are much stronger in the good rather than the bad channel, and increase 

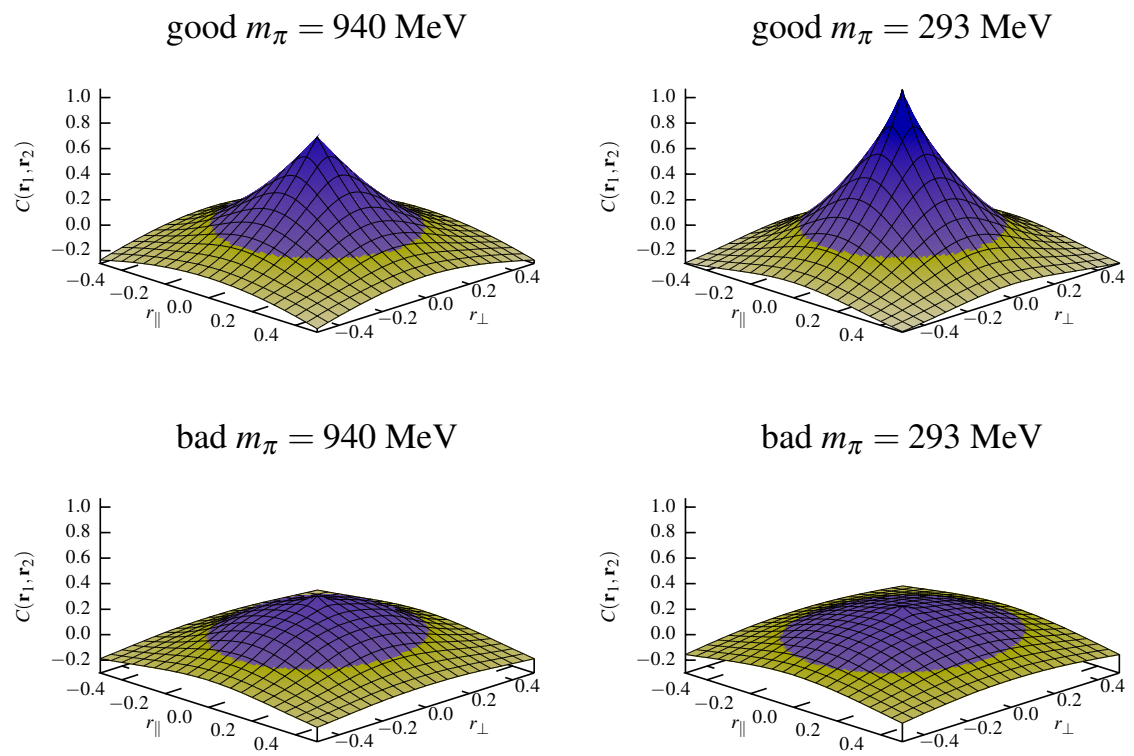

FIGURE 2. Continuous $C\left(\mathbf{r}_{1}, \mathbf{r}_{2}\right)$ derived from the fit, as a function of $\mathbf{r}$ (in fm) with $R=0.4 \mathrm{fm}$. The two axes $r_{\|}$and $r_{\perp}$ indicate directions of $\mathbf{r}$ parallel to and orthogonal to $\mathbf{R}$, respectively. The color of the surface is discontinuous at $C=0$.

strongly with decreasing quark mass. Finally, it is important to note that the diquark radius is approximately $0.3 \mathrm{fm}$ and the hadron half-density radius is also roughly $0.3 \mathrm{fm}$, so the diquark size is comparable to the hadron size. This is reminiscent of the size of Cooper pairs in nuclei, and argues against hadron models requiring point-like diquarks.

\section{ACKNOWLEDGMENTS}

This work was supported in part by funds provided by the U.S. Department of Energy under Grants No. DE-FG0294ER40818 and DE-FG02-96ER40965. P.V. acknowledges support by the MIT Undergraduate Research Opportunities Program (UROP). Additional domain wall propagators were computed using the Chroma software suite [15]. We also acknowledge the use of resources provided by the New Mexico Computing Applications Center (NMCAC) on Encanto, and of computer resources provided by the DOE through its support of the MIT Blue Gene/L.

\section{REFERENCES}

1. M. Anselmino, E. Predazzi, S. Ekelin, S. Fredriksson, and D. B. Lichtenberg, Rev. Mod. Phys. 65, 1199-1234 (1993).

2. I. C. Cloët, G. Eichmann, B. El-Bennich, T. Klähn, and C. D. Roberts, Few Body Syst. 46, 1-36 (2009), 0812 . 0416.

3. A. Selem, and F. Wilczek, "Hadron systematics and emergent diquarks," in New trends in HERA physics. Proceedings, Ringberg Workshop, Tegernsee, Germany, October 2-7, 2005, 2006, pp. 337-356, hep-ph/ 0602128.

4. R. L. Jaffe, Phys. Rept. 409, 1-45 (2005), hep-ph/ 0409065.

5. A. De Rújula, H. Georgi, and S. L. Glashow, Phys. Rev. D12, 147-162 (1975).

6. T. A. DeGrand, R. L. Jaffe, K. Johnson, and J. E. Kiskis, Phys. Rev. D12, 2060 (1975).

7. T. Schäfer, and E. V. Shuryak, Rev. Mod. Phys. 70, 323-426 (1998), hep-ph/ 9610451.

8. C. Alexandrou, P. de Forcrand, and B. Lucini, Phys. Rev. Lett. 97, 222002 (2006), hep-1at/ 0609004.

9. R. Babich, et al., Phys. Rev. D76, 074021 (2007), hep-lat/ 0701023.

10. J. Bratt, et al., Phys. Rev. D82, 094502 (2010), 1001.3620.

11. G. Kilcup, D. Pekurovsky, and L. Venkataraman, Nucl. Phys. Proc. Suppl. 53, 345-348 (1997), hep-lat/9609006.

12. C. Alexandrou, P. de Forcrand, and O. Jahn, Nucl. Phys. Proc. Suppl. 119, 667-669 (2003), hep-lat / 0209062.

13. T. T. Takahashi, H. Suganuma, Y. Nemoto, and H. Matsufuru, Phys. Rev. D65, 114509 (2002), hep-1at/ 0204011.

14. J. Green, M. Engelhardt, J. Negele, and P. Varilly, PoS LATTICE2010, 140 (2010), 1012.2353.

15. R. G. Edwards, and B. Joó, Nucl. Phys. Proc. Suppl. 140, 832 (2005), hep-lat/ 0409003. 